\title{
EFEITO DAS CONDIÇÕES OPERACIONAIS E DO TIPO DE SURFACTANTE SOBRE A ELETROSSÍNTESE DE FILMES $\mathrm{DE} \mathrm{PbO}_{2}$
}

\author{
R. M. FARINOS e L. A. M. RUOTOLO \\ Universidade Federal de São Carlos, Departamento de Engenharia Química \\ E-mail para contato: pluis@ufscar.br
}

\begin{abstract}
RESUMO - Eletrodos de $\mathrm{PbO}_{2}$ têm sido utilizados com sucesso para a oxidação de compostos orgânicos poluentes. Nestes processos, a morfologia e a aderência do filme de $\mathrm{PbO}_{2}$ são parâmetros importantes para evitar a liberação de chumbo para a solução e também para melhorar a eficiência da oxidação. Neste trabalho foi estudada a eletrossíntese galvanostática de $\mathrm{PbO}_{2}$ sobre um eletrodo de disco rotatório de grafite usando um planejamento fatorial fracionário $2^{4-1}$. O efeito da condição hidrodinâmica foi avaliado variando-se a velocidade de rotação do eletrodo. As outras variáveis estudadas foram a densidade de corrente, o tempo de síntese e a temperatura do eletrólito para três diferentes surfactantes. A morfologia e o recobrimento do substrato foram analisados por microscopia eletrônica de varredura. O poder eletrocatalítico dos eletrodos foi avaliado através da cinética de descoloração do corante azul reativo-19. Os resultados mostraram que a temperatura e o tipo de surfactante são as principais variáveis que afetam a qualidade e o desempenho eletrocatalítico do eletrodo formado.
\end{abstract}

\section{INTRODUÇÃO}

Atualmente existem diversas categorias de poluentes orgânicos gerados pelas atividades do homem moderno. Muitos destes poluentes são não biodegradáveis, necessitando, portanto, de tratamentos físico-químicos para sua degradação completa a $\mathrm{CO}_{2} \mathrm{e}$ $\mathrm{H}_{2} \mathrm{O}$ ou então para sua conversão em compostos orgânicos que sejam biodegradáveis.

Dentre os poluentes orgânicos intensivamente utilizados, os pesticidas ou defensivos agrícolas têm posição de destaque devido ao fato do Brasil ser um grande produtor de bens agrícolas, com o conseqüente consumo elevado destes tipos de compostos. As águas residuárias geradas tanto nos processos industriais de produção dos pesticidas quanto no processamento agrícola e manuseio de embalagens se constituem resíduos altamente tóxicos e devem ser adequadamente tratados para evitar a contaminação ambiental.

No estado de São Paulo, a cultura da laranja é de grande importância para a economia, o que fica evidente considerando-se o fato do Brasil ser o maior produtor mundial de cítricos e principal exportador de suco de laranja. Sendo assim, esta cadeia produtiva se constitui em uma grande consumidora de defensivos agrícolas, sendo que dentre os mais consumidos, segundo a Fundecitrus, estão o glifosato, o diuron e o tiametoxam. Diante deste cenário, torna-se imperativo que os efluentes gerados tanto no processo produtivo destes produtos 
quanto no manejo em campo destes pesticidas sejam adequadamente tratados antes de seu despejo em rios ou rede de esgoto.

Dentre os processos utilizados para o tratamento de poluentes orgânicos recalcitrantes destacam-se os Processos Oxidativos Avançados (POA's), os quais consistem na geração de radicais hidroxila $\left(\mathrm{HO}^{*}\right)$, para oxidação dos compostos orgânicos. Apesar de em muitos casos esses processos mostrarem-se eficientes para a oxidação de pesticidas, eles apresentam inconvenientes como o consumo de forma irreversível de produtos químicos e necessidade de sua armazenagem e, nos casos das técnicas mais elaboradas, há a dificuldade de ampliação de escala, além do custo de sua implementação. Nesse contexto, a busca por novos processos para o tratamento de efluentes contendo pesticidas tem se intensificado impulsionada muitas vezes pela necessidade de reuso da água dentro do próprio processo ou então para cumprir os padrões de emissão estabelecidos pela legislação ambiental vigente.

A tecnologia de oxidação eletroquímica de poluentes orgânicos oferece uma alternativa bastante versátil para o tratamento de efluentes aquosos contendo pesticidas uma vez que não envolve a utilização de produtos químicos, é de fácil manutenção e operação e o aumento de escala é bastante simples. Além de todos esses aspectos apontados, a tecnologia eletroquímica também se mostra ambientalmente compatível, uma vez que o principal "reagente" utilizado é o elétron.

Processos eletroquímicos, através da utilização de reatores eletroquímicos que empregam ânodos dimensionalmente estáveis (ADE's), são capazes de gerar radical hidroxila, fracamente adsorvidos, capazes de oxidar a matéria orgânica. Dentre os ânodos que fornecem excelentes resultados em termos de oxidação de moléculas orgânicas encontram-se aqueles que possuem um elevado sobrepotencial para a reação de desprendimento de oxigênio (RDO). Anodos de diamante dopado com boro (DDB), $\mathrm{SnO}_{2^{-}}$ $\mathrm{Sb}$ e $\mathrm{PbO}_{2}$ satisfazem esses critérios, porém o eletrodo de DDB, apesar de sua estabilidade química e ótimos resultados na degradação de compostos orgânicos, ainda possui um custo muito elevado, enquanto o eletrodo de $\mathrm{SnO}_{2}$-Sb desativa após algum tempo de uso (Montilla et al., 2004). Por outro lado, o eletrodo de $\mathrm{PbO}_{2}$, além de ser facilmente preparado por eletrodeposição, apresenta um baixo custo de produção e uma boa atividade eletroquímica, sendo estáveis em elevados potenciais aplicados e em meios com diferentes de pHs.

Apesar de todas as vantagens da tecnologia eletroquímica, sua principal desvantagem muitas vezes se deve ao elevado consumo de energia decorrente das baixas eficiências de corrente (Rajkumar et al., 2005), as quais, por sua vez, são conseqüência direta de restrições de transporte de massa ocasionadas pela baixa concentração do composto orgânico no efluente. Em problemas de remoção eletroquímica de íons metálicos presentes em soluções aquosas diluídas este problema é contornado pela utilização de cátodos tridimensionais (Britto-Costa e Ruotolo, 2009) que, além de uma elevada área superficial específica, proporcionam também altos valores de velocidade intersticial que geram turbulência e aumentam significativamente o coeficiente de transporte de massa. No entanto, para sistemas de tratamento eletroquímico de compostos orgânicos, a utilização de eletrodos tridimensionais é muitas vezes limitada pela escolha do material do substrato poroso a ser utilizado, o qual deve ser resistente à corrosão anódica, e também à dificuldade de deposição do material eletroativo sobre sua superfície. Recentemente, Recio et al. (2011) publicaram um artigo sobre a preparação de eletrodos de carbono vítreo reticulado recobertos com $\mathrm{PbO}_{2}\left(\mathrm{CVR} / \mathrm{PbO}_{2}\right)$, abrindo novas perspectivas para 
a aplicação deste eletrodo em tratamento de efluentes. Apesar dos resultados apresentados por Recio et al. (2011) ainda existe a necessidade de otimização das variáveis operacionais e do equipamento necessário para a realização da eletrossíntese do $\mathrm{PbO}_{2}$.

Neste trabalho foi estudado o efeito das condições hidrodinâmicas e de diferentes surfactantes sobre o filme de $\mathrm{PbO}_{2}$ sintetizado eletroquimicamente sobre um eletrodo de disco rotatório de grafite. Os filmes formados foram analisados quanto à sua morfologia através de microscopia eletrônica de varredura e sua atividade eletrocatalítica foi avaliada através da reação de descoloração do corante azul reativo AR-19.

\section{MATERIAIS E MÉTODOS}

Os filmes de $\mathrm{PbO}_{2}$ foram sintetizados galvanostaticamente sobre a superfície de um disco de grafite de $4 \mathrm{~mm}$ de diâmetro. O eletrólito usado na síntese era composto por $0,1 \mathrm{~mol}$ $\mathrm{L}^{-1}$ de $\mathrm{HNO}_{3}$ e 0,1 mol L${ }^{-1}$ de $\mathrm{Pb}\left(\mathrm{NO}_{3}\right)_{2}$. Três diferentes surfactantes foram adicionados ao eletrólito com a finalidade de se verificar sua influência sobre a morfologia e atividade eletrocatalítica do eletrodo. Esses surfactantes foram o laurilssulfato de sódio (LSS), o dodecilbenzenossulfonato de sódio (DBS) e Triton ${ }^{\circledR}$. Em todos os casos foi usada uma concentração de $0,5 \mathrm{mg} \mathrm{L}^{-1}$ de surfactante. $\mathrm{O}$ volume de eletrólito usado nas sínteses foi de $50 \mathrm{~mL}$.

Com a finalidade de se estudar o efeito da hidrodinâmica sobre as propriedades do filme utilizou-se um eletrodo de disco rotatório (Metrohm). A Figura 1 mostra o aparato experimental utilizado.

Estudou-se o efeito da densidade de corrente aplicada (i), da velocidade de rotação do eletrodo (w), do tempo de síntese (t) e da temperatura do eletrólito $(\mathrm{T})$ sobre as propriedades morfológicas e eletrocatalíticas dos filmes obtidos na presença dos diferentes surfactantes. Os efeitos destas quatro variáveis foram estudados segundo um planejamento fatorial de experimentos fracionário do tipo $2^{4-1}$ com três pontos centrais. A Tabela 1 mostra os níveis das variáveis, com suas codificações entre parênteses.
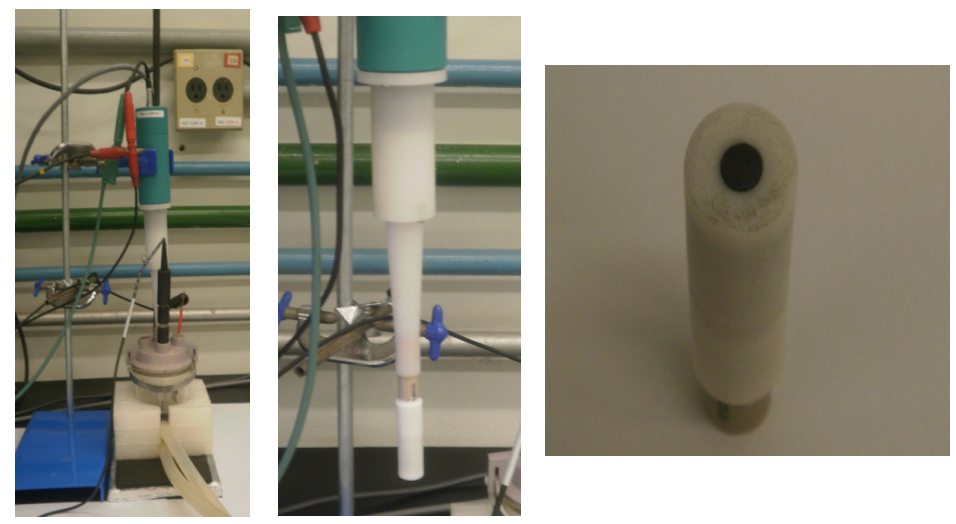

Figura 1 - Montagem experimental: (a) célula eletroquímica com controle da temperatura; (b) eletrodo de disco rotatório e (c) vista detalhada do eletrodo de grafite. 
Tabela 1 - Planejamento fatorial fracionário do tipo $2^{4-1}$.

\begin{tabular}{ccccc}
\hline Exp. & $\mathrm{i} / \mathrm{mA} \mathrm{cm}^{-2}$ & $\mathrm{w} / \mathrm{rad} \mathrm{s}^{-1}$ & $\mathrm{t} / \min$ & $\mathrm{T} /{ }^{\circ} \mathrm{C}$ \\
\hline 1 & $10(-1)$ & $20(-1)$ & $5(-1)$ & $40(-1)$ \\
2 & $30(+1)$ & $20(-1)$ & $5(-1)$ & $60(+1)$ \\
3 & $10(-1)$ & $200(+1)$ & $5(-1)$ & $60(+1)$ \\
4 & $30(+1)$ & $200(+1)$ & $5(-1)$ & $40(-1)$ \\
5 & $10(-1)$ & $20(-1)$ & $15(+1)$ & $60(+1)$ \\
6 & $30(+1)$ & $20(-1)$ & $15(+1)$ & $40(-1)$ \\
7 & $10(-1)$ & $200(+1)$ & $15(+1)$ & $40(-1)$ \\
8 & $30(+1)$ & $200(+1)$ & $15(+1)$ & $60(+1)$ \\
9 & $20(0)$ & $110(0)$ & $10(0)$ & $50(0)$ \\
10 & $20(0)$ & $110(0)$ & $10(0)$ & $50(0)$ \\
11 & $20(0)$ & $110(0)$ & $10(0)$ & $50(0)$ \\
\hline
\end{tabular}

A atividade eletrocatalítica do filme de $\mathrm{PbO}_{2}$ foi avaliada através do estudo da reação de descoloração do corante azul reativo 19 (AR-019). Nestes experimentos foi utilizado um volume de $50 \mathrm{~mL}$ de solução $30 \mathrm{mg} \mathrm{L}^{-1}$ de AR-19. As eletrólises foram realizadas aplicandose uma densidade de corrente de $50 \mathrm{~mA} \mathrm{~cm}{ }^{-2}$ durante 30 minutos e transcorrido este tempo procedia-se então à análise de absorbância em espectrofotômetro UV-Vis (Amersham Pharmacia) em comprimento de onda de $600 \mathrm{~nm}$.

A morfologia dos filmes foi analisada por microscopia eletrônica de varredura (MEV).

\section{RESULTADOS E DISCUSSÃO}

Primeiramente estudou-se o efeito das variáveis de síntese sobre a atividade eletrocatalítica do eletrodo obtido. Nestes experimentos, somente o surfactante LSS foi utilizado. A análise de raios-X do filme de $\mathrm{PbO}_{2}$ (Figura 2) mostra que os picos de difração indicam a presença de $\mathrm{PbO}_{2}$ na fase beta, o que é desejável considerando-se que esta é a fase mais ativa para aplicações em eletroxidação de compostos orgânicos.

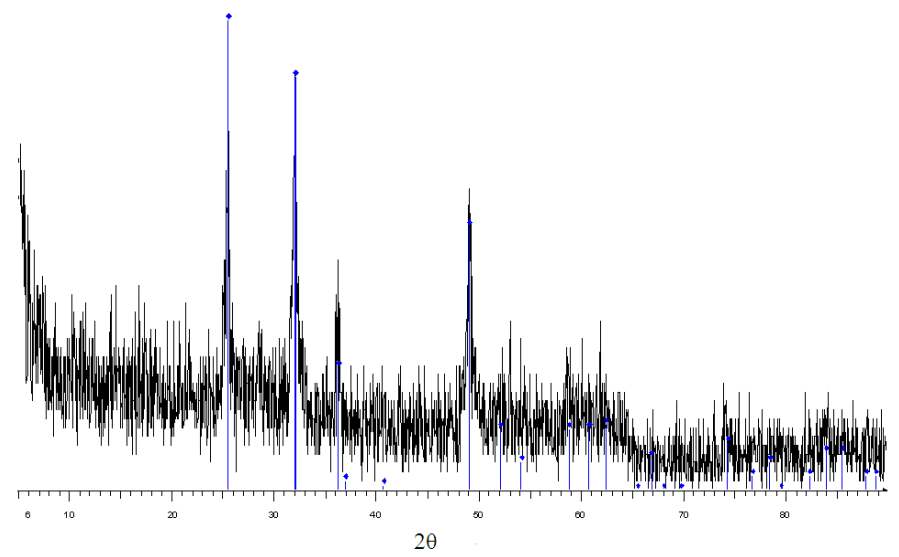

Figura 2: Difratograma de raios-X do filme de $\mathrm{PbO}_{2}$. 
A Figura 3 mostra o resultado da análise estatística para os testes eletrocatalíticos de descoloração do AR-19. O único efeito significativo sobre a porcentagem de descoloração do AR-19 (\%Descoloração), de acordo com o diagrama de Pareto da Figura 3(a), foi a temperatura de síntese. Assim sendo, de acordo com Figura 3b, uma temperatura de síntese elevada deve ser garantida de maneira a obter um eletrodo com elevada capacidade de eletroxidação.

A rotação do eletrodo não foi um fator determinante devido ao fato da concentração de $\mathrm{Pb}^{2+}$ na solução ser elevada, assim sendo, problemas com transferência de massa não limitam o crescimento do filme de $\mathrm{PbO}_{2}$. No caso da densidade de corrente e do tempo de síntese, acredita-se que eles não tiveram influência porque os valores mínimos de densidade de corrente e de tempo de síntese já seriam suficientes para a formação de um filme uniforme sobre a superfície do grafite. O uso de correntes e tempos maiores levaria apenas a um crescimento da espessura do filme. Como a reação eletroquímica ocorre apenas na superfície externa do filme de $\mathrm{PbO}_{2}$, o aumento da espessura do filme não levou a nenhuma melhoria no processo de descoloração.

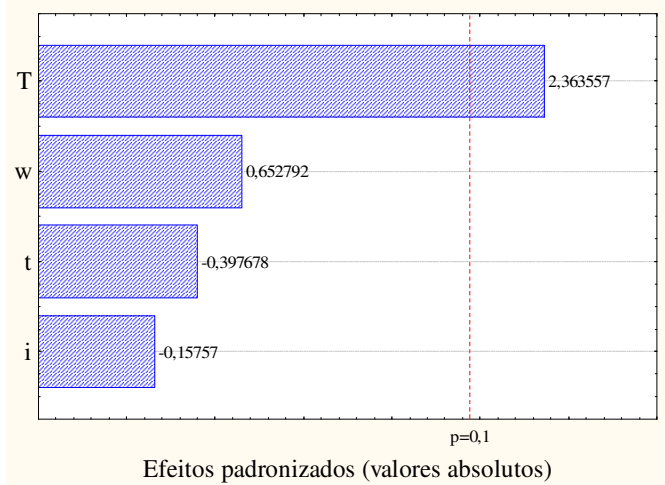

(a)

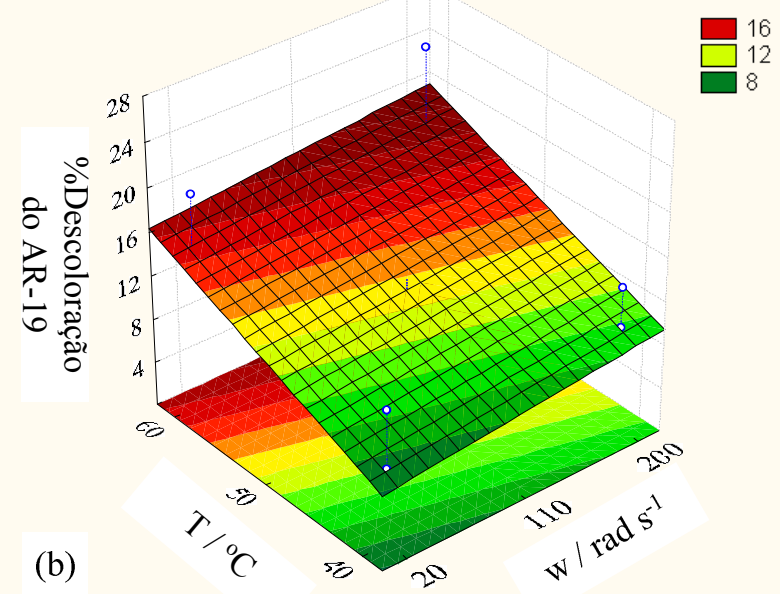

Figura 3- (a) Diagrama de Pareto dos efeitos e (b) porcentagem de descoloração em função da temperatura e da rotação do eletrodo.

Com a finalidade de se entender melhor o que estaria acontecendo, foram feitas imagens MEV dos eletrodos obtidos em meio de LSS. A Figura 4 mostra que a morfologia do depósito obtido depende bastante das condições de síntese e também da presença ou não do surfactante. $\mathrm{Na}$ ausência de surfactante, Figura 4(a), praticamente não se observa a presença do filme de $\mathrm{PbO}_{2}$ sobre a superfície do grafite, porém detectou-se a presença de $\mathrm{PbO}_{2}$ na forma de flocos no fundo do célula eletroquímica, indicando que houve a formação do óxido, porém ele não permaneceu aderido ao substrato. Por outro lado, quando se adicionou o LSS ao meio de síntese, houve a formação do filme de $\mathrm{PbO}_{2}$, no entanto, o depósito só ocorreu na temperatura de $60^{\circ} \mathrm{C}$, independentemente da velocidade de rotação utilizada. $\mathrm{O}$ filme obtido na temperatura de $60^{\circ} \mathrm{C}$ era bastante uniforme, com um recobrimento completo do substrato e apresentava a morfologia típica do $\beta-\mathrm{PbO}_{2}$, na forma de prismas. A espessura do filme formado era de aproximadamente $4 \mu \mathrm{m}$. 


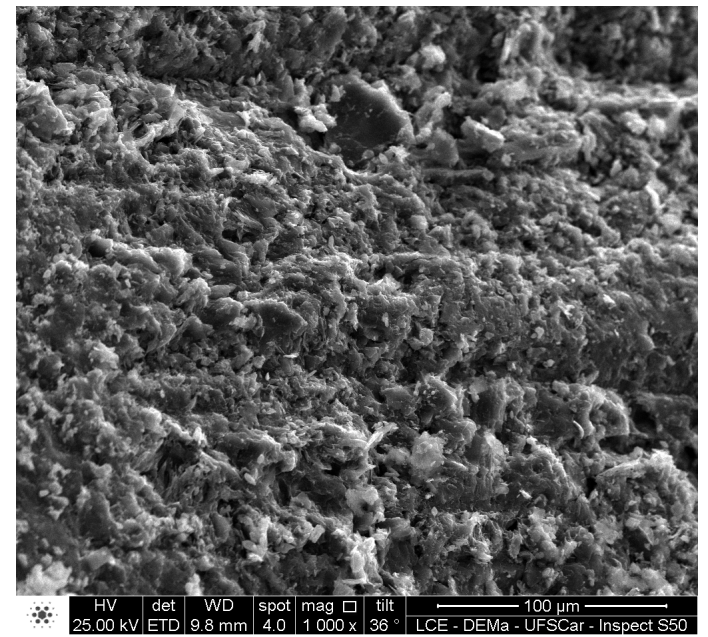

(a)

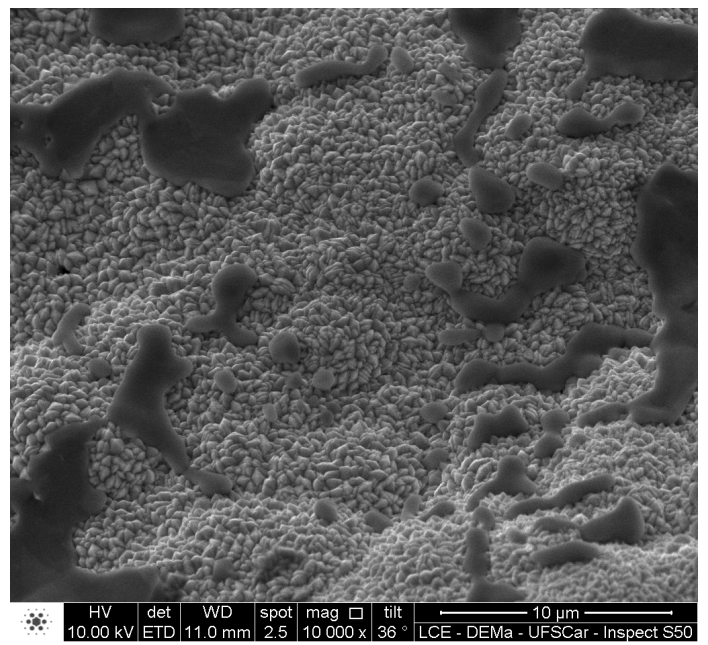

(c)

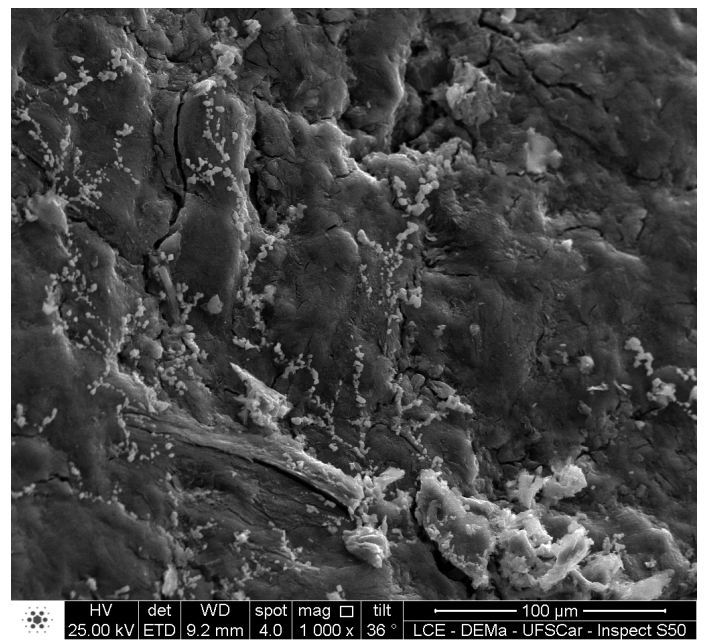

(b)

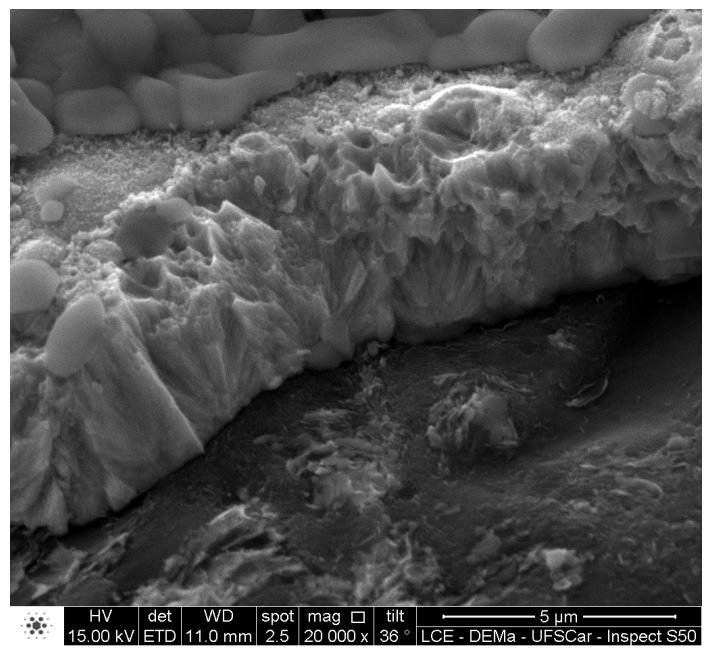

(d)

Figura 4 - Imagens MEV dos eletrodos obtidos na ausência de LSS (a), na presença de LSS com temperatura de $40^{\circ} \mathrm{C}$ (b) e na presença de LSS com temperatura de $60^{\circ} \mathrm{C}$, com aumentos de $10.000 X$ (c) e $20.000 X$ (d). $i=30 \mathrm{~mA} \mathrm{~cm}^{-2}$; w $=20 \mathrm{rad} / \mathrm{s}$.

Outros surfactantes também foram testados, como o DBS e o Triton ${ }^{\circledR}$. As imagens dos eletrodos obtidos para estes surfactantes são mostrados na Figura 5. No caso do DBS (Figura 5(a)), foi observado o mesmo comportamento verificado quando na ausência de surfactante, ou seja, detectou-se a presença de $\mathrm{PbO}_{2}$ na forma de flocos no fundo do célula eletroquímica. No entanto, utilizando-se o Triton ${ }^{\circledR}$, que é um surfactante líquido, observou-se a formação de $\mathrm{PbO}_{2}$ utilizando-se uma temperatura de $40^{\circ} \mathrm{C}$, porém verifica-se que o substrato não está totalmente recoberto pelo óxido.

Utilizando-se o surfactante Triton ${ }^{\circledR}$ observou-se uma mudança interessante no recobrimento do substrato uma vez que o depósito de $\mathrm{PbO}_{2}$ se apresentava na forma de uma teia em que porções do substrato podiam ser observadas. Especula-se que esta morfologia possa ser interessante para o processo de eletroxidação uma vez que o substrato de carbono 
poderia agir como sítios de adsorção da molécula orgânica que seriam então oxidadas pelos radicais hidroxila formados sobre o $\mathrm{PbO}_{2}$ nas adjacências.

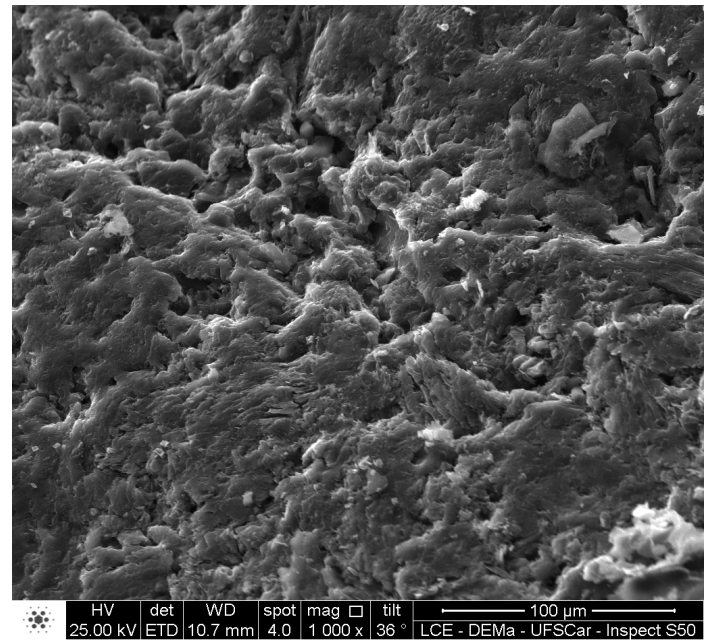

(a)

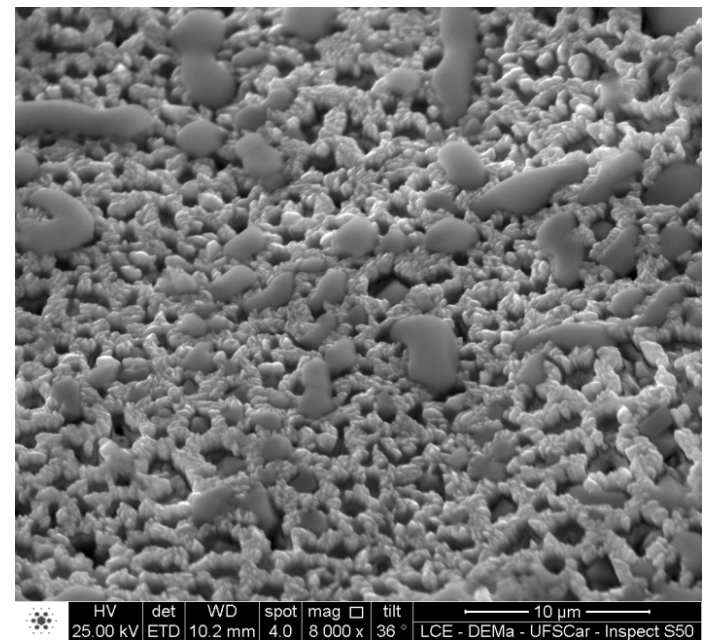

(b)

Figura 5 - Eletrodos obtidos utilizando-se DBS (a) e Triton $^{\circledR}$ (b). $i=30 \mathrm{~mA} \mathrm{~cm}^{-2}$; w $=20$ $\mathrm{rad} / \mathrm{s} ; \mathrm{T}=40^{\circ} \mathrm{C}$.

\section{CONCLUSÕES}

As principais conclusões do trabalho foram:

$\checkmark$ A presença e o tipo de surfactante são fundamentais para a obtenção de um depósito de $\mathrm{PbO}_{2}$ uniforme e aderente;

$\checkmark$ Utilizando-se o surfactante LSS, a temperatura de $60^{\circ} \mathrm{C}$ deve ser utilizada para a obtenção de um filme de $\mathrm{PbO}_{2}$ uniforme e aderente;

$\checkmark$ A corrente e o tempo de síntese devem ser suficientes para a formação de um filme que recubra toda a superfície do eletrodo;

$\checkmark$ Não se observou influência da hidrodinâmica (rotação do eletrodo) sobre o filme de $\mathrm{PbO}_{2}$ depositado.

\section{AGRADECIMENTOS}

Ao CNPq e à FAPESP pelo auxílio financeiro. 


\section{REFERÊNCIAS BIBLIOGRÁFICAS}

BRITTO-COSTA, P. H .Transferência de massa em Reator Eletroquímico de Carbono Vítreo Reticulado. Dissertação Mestrado- Universidade Federal de São de São Carlos, 2010.

MONTILlA, F.; MORALlÓN, E.; BATTISTI, A.; VÁSQUEZ, J. L. Preparation and Characterization of Antimony-Doped Tin Dioxide Electrodes. Part 1. Electrochemical characterization. J. Phys. Chem. B, v. 108, p. 5036-5043, 2004.

RAJKUMAR, D. ; KIM, J.G.;PALANIVELU,K. Indirect Electrochemical Oxidation of Phenol in the Presence of Chloride for Wastewater treatment, Chem. Eng. Technol., v. 28, p. 98, 2005.

RECIO, F.J.,; HERRASTI, P.; SIRÉS, I.; KULAK, A.N.; BAVYKIN, D.V.; PONCE-DELEON, C.; WALSH, F.C. The preparation of $\mathrm{PbO}_{2}$ coatings on reticulated vitreous carbon for the electro-oxidation of organic pollutants. Electrochim. Acta, v. 56, p. 5158$5165,2011$. 